\title{
Feasibility and Acceptability of Remote Physical Exercise Programs to Prevent Mobility Loss in Pre-Disabled Older Adults during Isolation Periods Such as the COVID-19 Pandemic
}

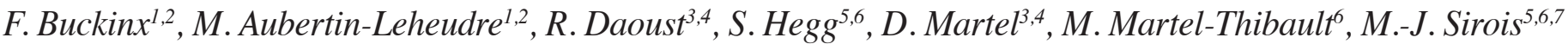

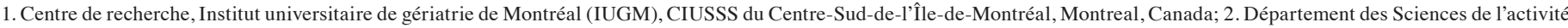

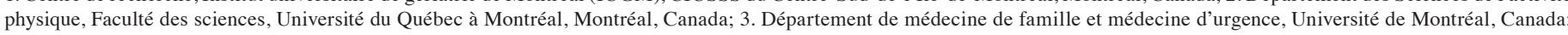

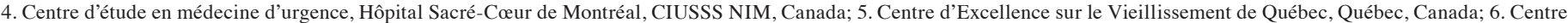 \\ de recherche du CHU de Québec-Université Laval, Québec, Canada; 7. Département de réadaptation, Université Laval, Québec, Canada
}

Corresponding Author: Marie-Josée Sirois, PhD, 1435 de Longueuil, Québec, Qc, G1S 2G2, Canada, e-mail: marie-josee.sirois@rea.ulaval.ca

\begin{abstract}
This study aimed to assess the feasibility and acceptability of remote physical exercise (PE) to prevent mobility loss among pre-disabled older adults during the COVID-19 lockdowns.

Participants followed a 12 -week PE remote program in Zoom(C) supervised groups (Web-Ex group, $\mathrm{n}=11$ ) or phone-supervised individual booklet-based home-program (Booklet group, $\mathrm{n}=33$ ).

The total rate of adherence was $82.5 \%$ in the Web-Ex group and $85.8 \%$ in the Booklet group. The level of satisfaction was « a lot » for $60 \%$ of the participants in the Web-ex group and for $37.9 \%$ of those included in the Booklet group. Respectively $10 \%$ and $31 \%$ of the participants rated the difficulty as « low » in the web-ex and Booklet groups.

Remote physical exercise using a web technology or booklets at home with regular and personalized follow-up during the lockdown was feasible and acceptable among pre-disabled seniors.
\end{abstract}

Key words: Remote exercise program, validation, adherence, satisfaction, difficulty, web technology.

\section{Background}

$\mathrm{N}$ ormal aging is often accompanied by a deterioration on functional abilities (1) that can be exacerbated by physical inactivity and sedentary lifestyle, which affect more than $50 \%$ of the older adults (2). In addition, this deterioration is associated with restricted mobility among older adults, creating a vicious cycle of deconditioning (2), which accelerates the spiral of loss of physical autonomy and increases the need for health care services (3). Social isolation that hit populations during the COVID-19 lockdowns, has accentuated the problem of inactivity and sedentary lifestyle (4). As such, sedentary time increased from 5 to 8 hours per day during COVID-19's first wave (5).

Fortunately, previous studies showed that pragmatic web tools integrating physical exercise (PE) programs that are adapted to older adults functional capacities (e.g. SPRINT (6) and MATCH (7) tools), are potential solutions to prevent their physical decline. A recent meta-analysis highlighted that home-based exercises appear effective to improve components of physical-fitness such as muscle strength, muscle endurance, muscle power, and balance (8). Moreover, according to a recent Received July 16, 2021 survey, $50 \%$ of seniors use the Internet every day and have a tablet, computer or smartphone (9). Thus, implementing remote PE using web technology could be a solution to maintain the health in older adults (10), while avoiding physical contact and risk of contagion. In that sense, Chaabene et al. concluded that, in times of restricted physical activity due to pandemics, homebased exercises are also an alternative to counteract physical inactivity and to keep older adults fit and healthy (8).

While the benefits of PA programs depend on continued participation (i.e. adherence), a change in lifestyle to include regular PE is difficult for many people of all ages. This is especially true in advanced ages (11) as increased co-morbidities, lack of social support, disability or depression in older adults may impede adherence rate to PA programs, which is particularly low in this population (12).

To date, few data related to the feasibility and acceptability of remote PE using web technology among seniors have been published. Thus, we aimed to assess the feasibility and acceptability of this type of intervention among pre-disabled older adults during isolation periods such as the COVID-19 pandemic.

\section{Methods}

\section{Study design and participants}

This is a 12-month intervention study, which started in May 2020 among 44 pre-disabled seniors who were previous participants of the Canadian "Cedecoms" trial (ClinicalTrial. gov: NCT03991598) testing the benefits of physical exercises after visiting the Emergency Department (ED) with a minor injury.

The trial inclusion criteria were: age $\geq 65$ years, presenting to ED with a minor injury not requiring hospital admission, independence in daily living activities (eating, toileting and transfers, dressing, showering, walking, continence) in the 4 weeks pre-injury, and discharged home after their ED assessment. Individuals who were hospitalized, living in a longterm care facility, unable to consent to the trial, not speaking English or French were excluded.

The current study is a convenience sample among 
participants included in the trial in 2019-20.

This research was approved by the REB of the "CHU de Québec-Université Laval" and all participants gave informed consent.

\section{Intervention}

Participants received a 12-week PE remote program (1 hour/3-times/week) in Zoom (C) supervised groups (Web-Ex group, $n=11$ ) or phone-supervised booklet-based individual home-program (booklet group, $n=33$ ). PE programs were adapted and specific to pre-disabled seniors, supervised by kinesiologist and each session (zoom $($ C or booklet) started with a 5-min warm-up, followed by $50 \mathrm{~min}$ of weight-bearing strengthening, standing balance, and light aerobic exercises, and ended with a 5-min cooldown including stretching.

\section{Measurements}

Participants were evaluated and interviewed by a trained kinesiologist every three months, through zoom $(\mathbb{C}$ meetings or by phone.

\section{Feasibility and acceptability of the intervention}

Based on the available literature, two indicators of the acceptability were measured (i.e. adherence rate and satisfaction) (13) while a proxy of feasibility was assessed in this study (i.e. the perceived difficulty) (14) : 1) Adherence rate: the proportion of completed week sessions out of the total week sessions prescribed (11). 2) Self-reported satisfaction: every two weeks of PE program, participants were asked to answer a 4-point likert scale $(0=$ not at all, $1=$ a little, $2=\operatorname{good}$, $3=\mathrm{a}$ lot) to assess their perceived level of satisfaction with the prescribed program. Then, the average of ratings over the 12-week duration of the program was calculated. 3) Perceived difficulty: every two weeks of PE program, participants were asked to answer a 4-point likert scale (i. 1=low, 2= moderate, $3=$ difficult, $4=$ very difficult) to assess their perceived level of difficulty with their program. Then, the average of ratings over the 12-week duration of the program was calculated.

In addition, qualitative interviews were conducted with the participants and the kinesiologists conducting the PE programs, in order to understand the facilitators and barriers to remote PE programs (web technology in groups or individual bookletbased programs). More specifically, participants were asked open-ended questions such as: "what do you think made it easy for you to get involved and to complete your PE program?", "What made it more difficult?", "What did you like or dislike about your PE program?" Questions were similar for the kinesiologists, e.g. "What did or did not facilitate conducting such remote PE programs and remote supervision of your participants?"

\section{Other measures}

The systematic review of Picorelli et al. highlighted personal factors associated with adherence to PE program (11). These confounding factors were therefore measured: Demographic factors: age, sex, main occupation, education (years of schooling completed), marital status. Health-related factors: perceived health (12-item Short-Form Health Survey: SF-12). Physical health factors: Body Mass Index, walking speed (timed 4-meter gait speed). Psychological factors: loneliness (UCLALoneliness Scale-3), cognitive status (Telephone Interview for Cognitive Status-TICS-m), fear of falling (FES-1 scale).

\section{Statistical analysis}

Data distributions were tested using the Kolmogorov test. Quantitative variables were expressed as means \pm standard deviations (SD). Qualitative variables were expressed as percentages. Independant t test were used to compare the means between groups, and Chi square tests were used to compare proportions. All statistical analyses were performed using SPSS 25.0 (Chicago, IL, USA). P-value $\leq 0.05$ was considered statistically significant.

The qualitative analyses consisted of reading each participant's responses in details and deductively categorizing the main ideas that emerged into themes. The small number of people interviewed did not require full transcriptions and coding into software such as QDA miner.

\section{Results}

\section{Participants}

Table 1 presents the baseline characteristics of the 44 participants included in the 12 -week intervention. At baseline, both groups were comparable regarding sociodemographic data, excepted for the level of education and the perceived general health which were higher in the Web-Ex group compared to the Booklet group $(\mathrm{p}=0.02$ and $\mathrm{p}=0.01$, respectively).

\section{Feasibility and acceptability of the intervention}

\section{Adherence rate}

The total adherence rate (proportion of completed week sessions out of the total week sessions prescribed) was $82.5 \%$ $(9.9 \pm 3.4 / 12$ weeks $)$ in the Web-Ex group and $85.8 \%(10.3 \pm$ $3.2 / 12$ weeks) in the Booklet group.

More specifically, in the Web-Ex group, $10 \%$ of the participants followed 2/12-week of the program, $10 \%$ followed 5-6/12 weeks, $20 \%$ followed 9-10/12 weeks and 60\% followed $11-12 / 12$ weeks. In the Booklet group, $3.3 \%$ of the participants followed 1/12-week of the program, $10 \%$ followed 3-4/12 weeks, $6.7 \%$ followed 7-8/12 weeks, $6.7 \%$ followed $9-10 / 12$ weeks and $73.3 \%$ followed 11-12/12 weeks (Figure 1). 
Table 1. Participants' baseline characteristics $(n=44)$

\begin{tabular}{|c|c|c|c|c|}
\hline & $\begin{array}{l}\text { Total participants } \\
\qquad(\mathrm{n}=44)\end{array}$ & $\begin{array}{l}\text { Web-Ex group } \\
\quad(n=11)\end{array}$ & $\begin{array}{l}\text { Booklet group } \\
\qquad(\mathrm{n}=33)\end{array}$ & p-value \\
\hline \multicolumn{5}{|l|}{ Socio-demographic factors } \\
\hline Sex (women) & $30(68.1)$ & $8(72.7)$ & $22(71)$ & 0.91 \\
\hline Age (years) & $79.3 \pm 6.2$ & $77 \pm 6.9$ & $80.1 \pm 5.9$ & 0.41 \\
\hline Main occupation & & & & 0.55 \\
\hline - Full- or part- time work & $1(2.3)$ & $0(0)$ & $1(3.03)$ & \\
\hline - Retirement & $41(93.2)$ & $10(90.9)$ & $31(93.4)$ & \\
\hline - Volunteer work & $3(6.8)$ & $1(3.03)$ & $2(18.2)$ & \\
\hline Education & & & & 0.02 \\
\hline - Primary school & $5(11.3)$ & $0(0)$ & $5(16.1)$ & \\
\hline - High school & $8(18.2)$ & $2(18.2)$ & $6(19.4)$ & \\
\hline - College & $14(31.8)$ & $3(27.3)$ & $11(35.4)$ & \\
\hline - University & $15(34.1)$ & $6(54.6)$ & $9(29)$ & \\
\hline Marital status & & & & 0.20 \\
\hline - Married & $18(40.9)$ & $5(45.4)$ & $13(41.9)$ & \\
\hline - Living with partner & $2(4.5)$ & $1(9.09)$ & $1(3.23)$ & \\
\hline - Divorced/separated & $6(13.6)$ & $0(0)$ & $6(19.4)$ & \\
\hline - Widowed & $11(25)$ & $2(18.2)$ & $9(23.0)$ & \\
\hline - Single, never married & $5(11.4)$ & $3(27.3)$ & $2(6.45)$ & \\
\hline \multicolumn{5}{|l|}{ Health related status } \\
\hline Perceived general health: SF-12 & & & & 0.01 \\
\hline - Excellent & $1(2.2)$ & $1(9.2)$ & $0(0)$ & \\
\hline - Very good & $24(54.5)$ & $3(29.9)$ & $21(61.8)$ & \\
\hline - Good & $12(27.3)$ & $4(36.8)$ & $8(23.5)$ & \\
\hline - Fair & $6(13.6)$ & $2(18.4)$ & $4(11.8)$ & \\
\hline - Poor & $0(0)$ & $0(0)$ & $0(0)$ & \\
\hline \multicolumn{5}{|l|}{ Physical factors } \\
\hline $\operatorname{BMI}\left(\mathrm{kg} / \mathrm{m}^{2}\right)$ & $27.5 \pm 5.0$ & $25.8 \pm 1.8$ & $28.0 \pm 6.1$ & 0.54 \\
\hline Physical function: 4m- gait speed (m/s) & $5.5 \pm 1.6$ & $5.9 \pm 1.9$ & $5.5 \pm 1.9$ & 0.79 \\
\hline \multicolumn{5}{|l|}{ Psychological factors } \\
\hline Loneliness : UCLA score (/9) & $4.3 \pm 1.6$ & $3.8 \pm 1.3$ & $4.3 \pm 1.4$ & 0.11 \\
\hline Cognitive status : TICS (/50) & $36.9 \pm 9.1$ & $34.9 \pm 12.7$ & $34.8 \pm 10.1$ & 0.31 \\
\hline Fear of falling : FES-I (/28) & $4.4 \pm 4.1$ & $3.6 \pm 4.4$ & $4.4 \pm 4.1$ & 0.06 \\
\hline
\end{tabular}

Legend : SF-12: 12-item Short Form Survey; BMI:Body Mass Index ; TICS: Telephone Interview for Cognitive Status; FES-I : Falls Efficacy Scale International

During the 12-week intervention, 7 participants dropped out: 5 in the Booklet group (16\%, including 1 due to COVID-19 positive) and 2 in the WEB-Ex group (18\%).

\section{Self-reported satisfaction}

On average (mean on 12-week PA program), 4 participants $(40 \%)$ in the Web-Ex group expressed «good » satisfaction with their PE program, while 7 (60\%) were « a lot» satisfied. In the Booklet group, the level of the satisfaction was « a little» (1 participant (3.5\%)), « good» (19 participants (58.6\%)) and « a lot $»$ for 13 of them (37.9\%). In addition, as shown in Figure
2, the level of satisfaction remained constant throughout the intervention period in the Web-Ex group while it has improved in the Booklet group in which the proportion of subjects who were satisfied «a lot» with the PE program increased between the first (W1) and the 12th week (W12). Conversely, the proportion of subjects with «good» satisfaction decrease between W1 and W12.

\section{Perceived difficulty}

In the Web-Ex group, 10 participants estimated the overall difficulty of their PE program with 9 of them (90\%) rating it 
as « moderate » and $1(10 \%)$ rating it as « low ». Interestingly, at W1, 4 participants (40\%) rated the difficulty as « low » and $6(60 \%)$ as « moderate », but at W12, 3 participants $(33.3 \%)$ rated the difficulty as « low », $6(55.6 \%)$ as « moderate » and 1 $(11.1 \%)$ as « difficult ».

In the Booklet group, 29 participants estimated the difficulty of the PE program. Among them, 20 (69\%) rated the difficulty as « moderate », while $9(31 \%)$ rated it as « low ». Similarly to the other group, at W1, 10 participants $(36 \%)$ of the Booklet group rated the difficulty as « low », $17(57 \%)$ as « moderate» and $2(7 \%)$ as « difficult » but at W12, 8 participants $(29 \%)$ rated the difficulty as « low» and $21(71 \%)$ as «moderate ».

\section{Figure 1. Adherence rate to the intervention, by group}

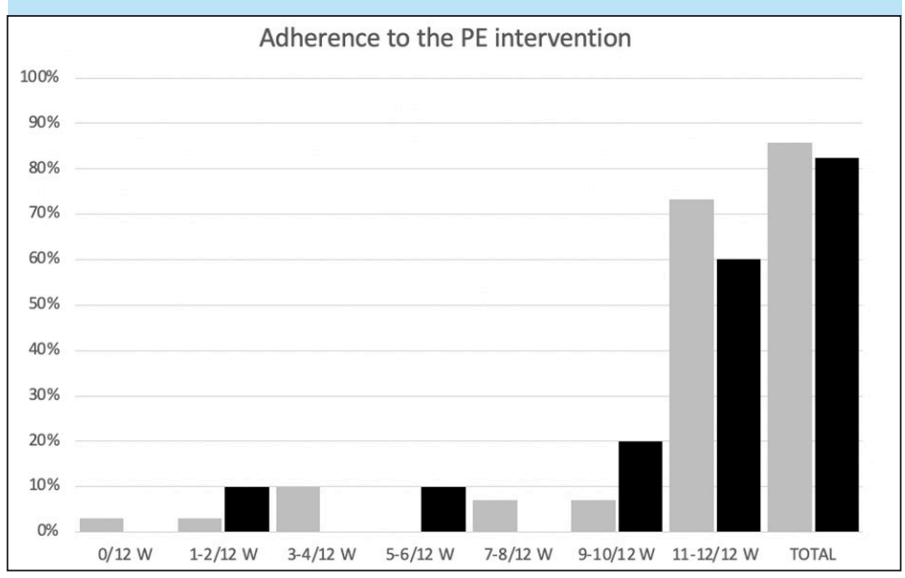

Legend: $\mathrm{W}=$ weeks

\section{Barriers and facilitators}

The participants from the Booklet group identified the following facilitators to their remote PE program: the flexible schedule to perform physical activity sessions, the individual online follow-up and the continuity of services from the kinesiologists throughout the intervention. The participants from the Web-Ex group noted the following facilitators to their remote PE program: the motivation generated by group training, and by the novelty (i.e. new experience), the regular help and follow-up from the kinesiologists, and the fixed and prepredetermined schedule.

From the investigators point of view, the facilitators to remote PE using web technology were: no need to travel, the motivation to try something new, the possibility of maintaining interactions and exercigesing with patients despite the COVID-19 lockdown, remaining available and accessible between sessions for questions or problem solving, sending programs (Booklet group) according to participants' preferred mean (at the door step, email or regular mail) and real time adaptation and feedback for Web-ex participants. In addition, kinesiologists reported that their constant professional commitment made participants feel they were taking care of and helped maintain their adherence to PE programs.

Participants and kinesiologists reported both barriers to remote $\mathrm{PE}$ to be linked to 1) the technology itself: variety of systems (PC, MAC, tablets, computers), difficulty of understanding and using technology devices, need for teaching technology to older adults, and to 2) the clinical aspects: test setup and safety, difficulty to provide remote assistance, to see the whole body during zoom sessions and to provide feedback on exercises as well as difficulty to perceive pain and restrictions or injury (feeling of insecurity).

\section{Discussion}

This study aimed to assess the feasibility (adherence rate and self-reported satisfaction) and acceptability (perceived difficulty) of remote PE among pre-disabled older adults to preserve health during isolation periods such as the COVID-19 pandemic.

A high adherence rate to PE program above $80 \%$ in both intervention groups was observed, suggesting that pre-disabled older adults are capable of sustaining PE for 12 weeks. These results are better that the 58 to $77 \%$ average adherence rates previously reported among older adults 11 and could potentially be explained by the participants' socio-demographic characteristics. As indicated by Picorelli et al., higher socioeconomic status and living alone are associated with better adherence to PE program 11 along with presenting fewer health conditions, better self-rated health, better physical and cognitive abilities and fewer depressive symptoms 11. Although our participants were pre-disabled older adults, they shared many favorable characteristics to good adhesion (see Table 1). As mentioned in the qualitative interviews, even if remote, active communication and personalized monitoring by the kinesiologists (health specialist in exercise) contributed to our high adherence rates 15 .

With regards to satisfaction with $\mathrm{PE}$ programs, there is evidence suggesting that satisfaction is a critical enabling factor for older people to engage in PE 16. With $95 \%$ of our participants from both intervention groups expressed at least good satisfaction with their PE programs, it is very likely that satisfaction contributed to the high adherence rate we observed.

Our results also indicate that the perceived level of difficulty was low to moderate in both intervention groups and the adherence rate was high, suggesting that the prescribed PE programs were acceptable. Our findings are in line with previous studies demonstrating that home-based exercise technology is acceptable in various population such as community-dwelling older adults who sustained a minor injury 17, nursing home residents with and without mild cognitive impairments 18 but also among pre-disabled older adults 19 .

Besides the professional follow-up mentioned above, another important facilitator to remote PE intervention that emerged from our study, from both investigators and participant's point of view, is the motivation to try something new. We can hypothesize that the investigators motivated by the study helped to motivate participants to take part and adhere to the intervention. According to a publication understanding motivations to participate in a research study, the role of the investigators is important. Effectively, several participants 
Figure 2. Levels of satisfaction in participants during the 12-week intervention, according to groups (Figure 2A : Booklet group; Figure 2B: Web-Ex group)

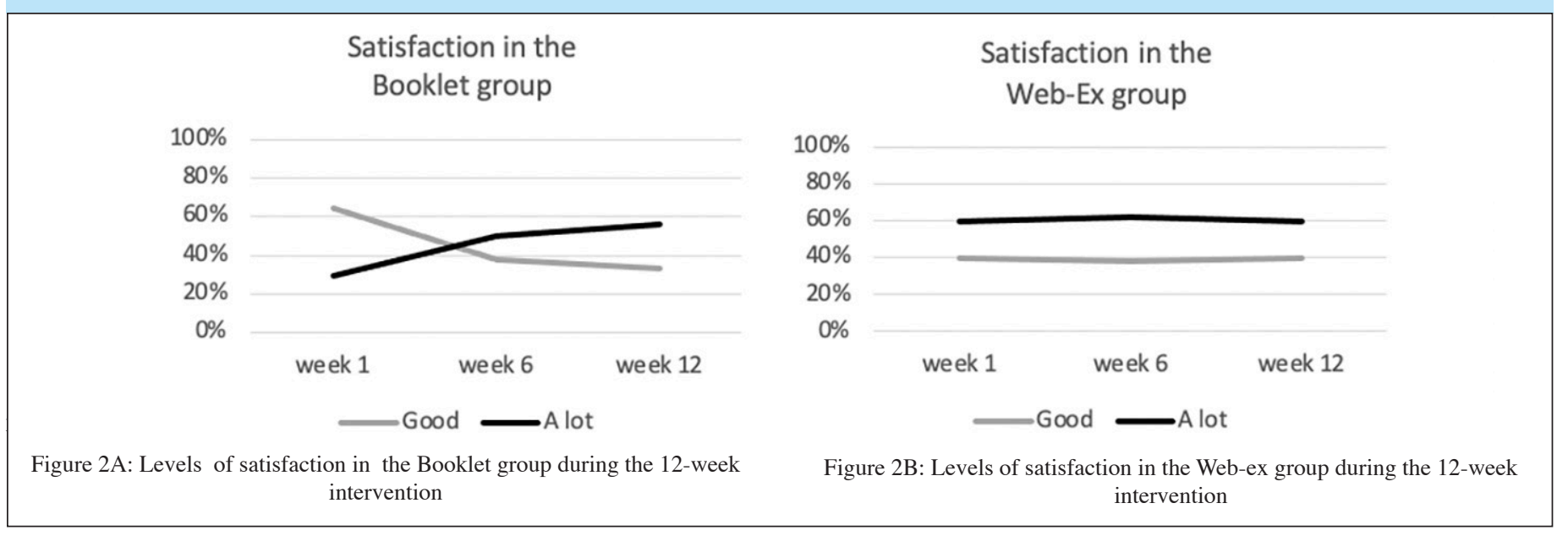

participate in the study 20. Understanding of motivational factors for participation in and adherence to, exercise programmes is of great importance to older people, health professionals and society as more than $50 \%$ of older adults are inactive or sedentary.

Despite our results add to a growing literature focused on investigating remote $\mathrm{PE}$ interventions, some potential bias and limitations must be acknowledged. First, a selection bias is possible because of the selection criteria for this study (e.g. volunteer subjects, access to a computer or tablet or smartphone). Because only pre-disabled older adults were included, participants are not representative of the general older adults population. The small sample size also limits the statistical power and the generalizability of our conclusion to larger older adults populations. Then, a social desirability bias is possible since participants knew they were being assessed throughout the study. In addition, we have a potential measurement bias because of the online modality. Effectively, online assessments could affect the reliability and reproducibility of the tests (greater margin of error measurements, viewing angle, lack of postural control, response times from participants). Finally, an information bias is possible since date were self-reported. The judgment of the investigators plays a role in participants' responses.

\section{Conclusion}

Remote physical exercise using web technology or booklets with regular and personalized follow-ups during the COVID-19 lockdowns seemed feasible and acceptable among pre-disabled seniors. The adherence rate was high in both modalities. However, the level of satisfaction was higher in the webex group while the perceived difficulty was higher in the booklet one. Both interventions web technology or booklet with regular and personalized follow-up could help to prevent deconditioning and loss of autonomy in older adults while allowing social interaction during a lockdown period.
Funding: This study was funded by the «Fonds de Recherche du Québec -Santé (FRQS)», by the «QUebec Network for Research on Aging» (RQRV) and by the Canadian Institutes for Health Research (competition: 201603PJT, application \#: 364485). Fanny Buckinx is supported by the «Fonds de Recherche du Québec Santé (FRQS) »

Acknowledgments: Authors wish to thank Mr Pierre-Hugues Carmichael, MSc, statistician at the Centre d'Excellence sur le Vieillissement de Québec (CEVQ).

Conflicts of interest: The authors have no conflicts of interest to declare

Ethical standards: All procedures performed in accordance with the ethical standards of the institutional research committee and with the 1964 Helsinki declaration and its later amendments or comparable ethical standards.

Open Access: This article is distributed under the terms of the Creative Commons Attribution 4.0 International License (http://creativecommons.org/licenses/by/4.0/), which permits use, duplication, adaptation, distribution and reproduction in any medium or format, as long as you give appropriate credit to the original author(s) and the source, provide a link to the Creative Commons license and indicate if changes were made.

\section{References}

1. Bouchard DR, Janssen I. Dynapenic-obesity and physical function in older adults. The journals of gerontology Series A, Biological sciences and medical sciences. Jan 2010;65(1):71-7. doi:10.1093/gerona/glp159

2. Cunningham C, R OS, Caserotti P, Tully MA. Consequences of physical inactivity in older adults: A systematic review of reviews and meta-analyses. Scandinavian journal of medicine \& science in sports. May 2020;30(5):816-827. doi:10.1111/sms.13616

3. Ding D, Lawson KD, Kolbe-Alexander TL, et al. The economic burden of physical inactivity: a global analysis of major non-communicable diseases. Lancet (London, England). Sep 24 2016;388(10051):1311-24. doi:10.1016/s0140-6736(16)30383-x

4. Aubertin-Leheudre M, Rolland Y. The Importance of Physical Activity to Care for Frail Older Adults During the COVID-19 Pandemic. Journal of the American Medical Directors Association. Jul 2020;21(7):973-976. doi:10.1016/j.jamda.2020.04.022

5. Chambonniere C, Lambert C, Tardieu M, et al. Physical Activity and Sedentary Behavior of Elderly Populations during Confinement: Results from the FRENCH COVID-19 ONAPS Survey. Exp Aging Res. Apr 7 2021:1-13. doi:10.1080/036107 3x.2021.1908750

6. Peyrusqué E, Buckinx F, Bolduc A, Law C, Kergoat MJ, Aubertin-Leheudre M. Potential Efficacy of Pragmatic Exercise Program (SPRINT) during Hospitalization in Older Adults on Health Care and Physical Performance: A Pilot Study. The journal of nutrition, health \& aging. 2021;25(1):126-133. doi:10.1007/s12603-020-1483-4

7. Peyrusqué E, Kergoat MJ, Bolduc A, et al. Maintenance of Autonomy Through exerCise in Hospital Setting (MATCH): A Feasibility Study. Journal of the American Medical Directors Association. Apr 2021;22(4):873-875. doi:10.1016/j. jamda.2020.12.043

8. Chaabene H, Prieske O, Herz M, et al. Home-based exercise programmes improve physical fitness of healthy older adults: A PRISMA-compliant systematic review and meta-analysis with relevance for COVID-19. Ageing research reviews. May 2021;67:101265. doi:10.1016/j.arr.2021.101265

9. Brittne NK. 2020 Tech Trends of the 50+. AARP Research. January 2020 2020; doi:https://doi.org/10.26419/res.00329.001

10. Barbosa Neves B, Franz R, Judges R, Beermann C, Baecker R. Can Digital 
Technology Enhance Social Connectedness Among Older Adults? A Feasibility Study. Journal of applied gerontology : the official journal of the Southern Gerontological Society. Jan 2019;38(1):49-72. doi:10.1177/0733464817741369

11. Picorelli AM, Pereira LS, Pereira DS, Felício D, Sherrington C. Adherence to exercise programs for older people is influenced by program characteristics and personal factors: a systematic review. Journal of physiotherapy. Sep 2014;60(3):151-6 doi:10.1016/j.jphys.2014.06.012

12. Dolansky MA, Stepanczuk B, Charvat JM, Moore SM. Women's and men's exercise adherence after a cardiac event. Research in gerontological nursing. Jan 2010;3(1):308. doi:10.3928/19404921-20090706-03

13. Andrews G, Basu A, Cuijpers P, et al. Computer therapy for the anxiety and depression disorders is effective, acceptable and practical health care: An updated meta-analysis. Journal of anxiety disorders. Apr 2018;55:70-78. doi:10.1016/j.janxdis.2018.01.001

14. Bowen DJ, Kreuter M, Spring B, et al. How we design feasibility studies. American journal of preventive medicine. May 2009;36(5):452-7. doi:10.1016/j. amepre.2009.02.002

15. Feng NC, Ryan E, Kidane M, et al. Feasibility of an at-home, web-based, interactive exercise program for older adults. Alzheimer's \& dementia (New York, N Y) 2019;5:825-833, doi:10.1016/j.trci.2019.10.005
16. Devereux-Fitzgerald A, Powell R, Dewhurst A, French DP. The acceptability of physical activity interventions to older adults: A systematic review and metasynthesis. Social science \& medicine (1982). Jun 2016;158:14-23. doi:10.1016/j. socscimed.2016.04.006

17. Lauze M, Martel DD, Agnoux A, et al. Feasibility, Acceptability and Effects of a Home-Based Exercise Program Using a Gerontechnology on Physical Capacities after a Minor Injury in Community-Living Older Adults: A Pilot Study. The journal of nutrition, health \& aging. 2018;22(1):16-25. doi:10.1007/s12603-017-0938-8

18. Buckinx F, Waters DL, Aubertin-Leheudre M. Letter to the Editor: Implementing Home-Based Exercise Technology in a Nursing Home: Does MCI Status Matter? The Journal of frailty \& aging. 2021;10(1):77-78. doi:10.14283/jfa.2020.34

19. Valiani V, Lauze M, Martel D, et al. A New Adaptive Home-based Exercise Technology among Older Adults Living in Nursing Home: A Pilot Study on Feasibility, Acceptability and Physical Performance. The journal of nutrition, health \& aging. 2017;21(7):819-824. doi:10.1007/s12603-016-0820-0

20. Soule MC, Beale EE, Suarez L, et al. Understanding motivations to participate in an observational research study: Why do patients enroll? Social work in health care 2016;55(3):231-46. doi:10.1080/00981389.2015.1114064

How to cite this article: F. Buckinx, M. Aubertin-Leheudre, R. Daoust, et al. Feasibility and Acceptability of Remote Physical Exercise Programs to Prevent Mobility Loss in Pre-Disabled Older Adults during Isolation Periods Such as the COVID-19 Pandemic. J Nutr Health Aging. 2021;25(9):1106-1111, http://dx.doi.org/10.1007/s12603-021-1688-1 